What ethologically based models have taught us about the neural systems underlying fear and anxiety

N.S. Canteras, S.R. Mota-Ortiz and S.C. Motta

The Brazilian Journal of Medical and Biological Research is partially financed by

\section{惡CNPq}

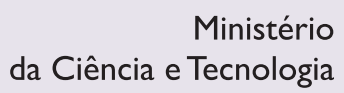

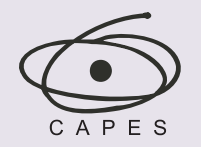

Ministério da Educação
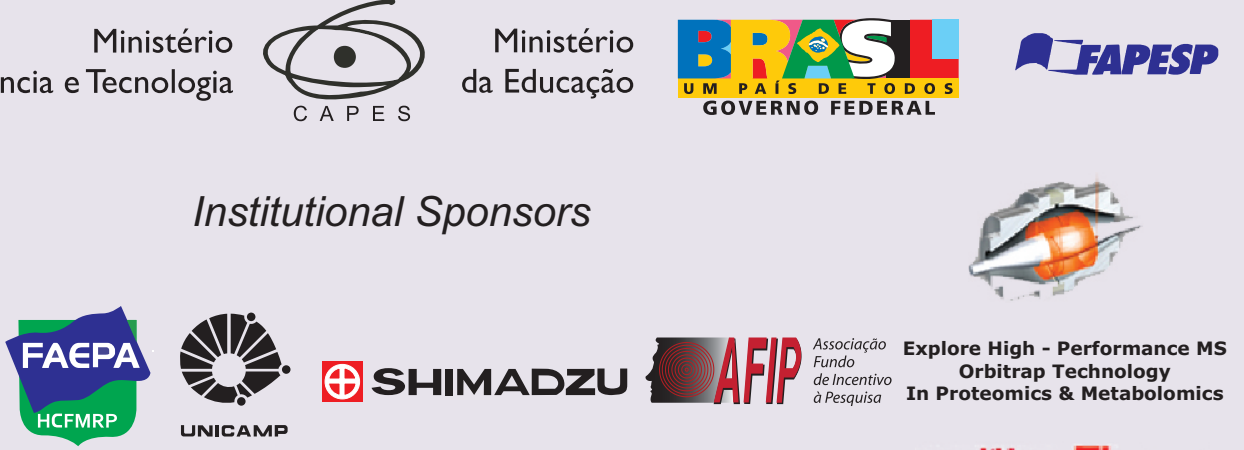

Institutional Sponsors

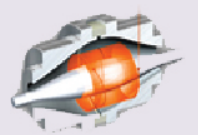

Ф SHIMADZU UNICAMP

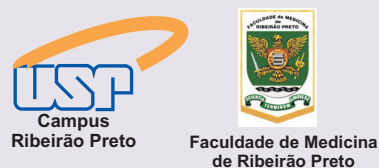

de Ribeirão Preto
de 


\title{
What ethologically based models have taught us about the neural systems underlying fear and anxiety
}

\author{
N.S. Canteras ${ }^{1}$, S.R. Mota-Ortiz ${ }^{2}$ and S.C. Motta ${ }^{1}$ \\ 1Departamento de Anatomia, Instituto de Ciências Biomédicas, \\ Universidade de São Paulo, São Paulo, SP, Brasil \\ 2Laboratório Bases Neurais do Comportamento, \\ Universidade Cidade de São Paulo, São Paulo, SP, Brasil
}

\begin{abstract}
Classical Pavlovian fear conditioning to painful stimuli has provided the generally accepted view of a core system centered in the central amygdala to organize fear responses. Ethologically based models using other sources of threat likely to be expected in a natural environment, such as predators or aggressive dominant conspecifics, have challenged this concept of a unitary core circuit for fear processing. We discuss here what the ethologically based models have told us about the neural systems organizing fear responses. We explored the concept that parallel paths process different classes of threats, and that these different paths influence distinct regions in the periaqueductal gray - a critical element for the organization of all kinds of fear responses. Despite this parallel processing of different kinds of threats, we have discussed an interesting emerging view that common cortical-hippocampal-amygdalar paths seem to be engaged in fear conditioning to painful stimuli, to predators and, perhaps, to aggressive dominant conspecifics as well. Overall, the aim of this review is to bring into focus a more global and comprehensive view of the systems organizing fear responses.
\end{abstract}

Key words: Fear; Predator exposure; Social threat; Fear memory; Contextual fear; Hypothalamus

\section{Introduction}

The prevailing view of central fear system organization emerged from studies using classical Pavlovian fear conditioning, indicating the amygdala as a major player in learning, storage, and expression of fear responses (1). Among the amygdalar regions, two nuclei have been particularly focused upon in fear-conditioning research, namely, the lateral and central nuclei. The lateral nucleus of the amygdala is thought to be the primary site where associations between the conditioned stimulus (CS) and the unconditioned stimulus (US) are formed and stored, and is known as a critical site for fear memory (2). Conversely, the central nucleus, via projections to the hypothalamus and brainstem, is critical for the expression of fear conditioning. In fact, lesions of the central nucleus disrupt freezing, along with the autonomic reactions observed during fear conditioning (1). More recent studies, however, have also suggested a role for the central nucleus in learning and storage of fear conditioning (3). However, the view of a unitary central fear system has been challenged by several experimental lines of evidence, and lesions of the central nucleus have been found to have no effect on either unconditioned or conditioned defensive responses to a live predator or its odor $(4,5)$.

The use of ethologically based threats, like predator exposure and attack by conspecifics, has provided an interesting prospective on how fear responses should be organized by parallel circuits involving the amygdala and medial hypothalamic nuclei.

In this article, we reviewed how different threats are processed by these parallel circuits, likely to be preserved across species, and how they target the periaqueductal gray (PAG), a critical brain site for the organization of fear responses. Moreover, we also analyzed the pathways involved in fear memory for different kinds of threats, high-

Correspondence: N.S. Canteras, Departamento de Anatomia, ICB, USP, Av. Professor Lineu Prestes, 2465, 05508-000 São Paulo, SP, Brasil. E-mail: newton@icb.usp.br

Presented at the III Fórum em Neurobiologia do Estresse, Araraquara, SP, Brazil, September 8-10, 2011.

Received December 20, 2011. Accepted March 9, 2012. Available online March 30, 2012. Published April 9, 2012. 
lighting the emerging view that different fear memories may indeed share common cortical-hippocampal-amygdalar paths. The aim of this article is to bring into focus a more global and comprehensive view of the systems organizing fear responses, challenging the concept of a unitary core circuit for fear processing, and yet providing the communalities among the fear memory circuits.

\section{Different classes of threats mobilize distinct neural systems}

The medial zone of the hypothalamus has been shown to have a critical role in processing threatening stimuli, like predator exposure and social threats from dominant conspecifics, which, under natural circumstances, are the events more likely to evoke the sensation of fear and the accompanying defensive responses $(6,7)$.

A systematic analysis of axonal projections from the medial nuclei of the hypothalamus indicates that they form two partially segregated circuits respectively underlying the expression of reproductive and defensive behaviors. The overall results regarding the axonal projections of the medial hypothalamic zone indicate that the anterior hypothalamic nucleus, the dorsomedial part of the ventromedial nucleus, and the ventrolateral part of the dorsal premamillary nucleus are extensively interconnected (the predator-responsive medial hypothalamic circuit), and are partially segregated from another medial zone circuit that includes the medial preoptic nucleus and the ventrolateral part of the ventromedial, tuberal, and ventral premamillary nuclei (the social/ reproductive medial hypothalamic circuit) (8).

Exposure to a predator or its odor has been shown to up-regulate c-fos expression in the circuit formed by the anterior hypothalamic nucleus, the dorsomedial part of the ventromedial nucleus, and the ventrolateral part of the dorsal premamillary nucleus (PMD) - the predator-responsive medial hypothalamic circuit (Figure 1) (6). In rodents, the dorsal premamillary nucleus, particularly its ventrolateral part, represents the most sensitive brain region responding to a predator or its clues. In fact, the dorsal premammillary nucleus appears to work as an amplifier for this medial hypothalamic circuit processing predator cues. This would explain why this region is so responsive to predator threats, and why lesions therein are able to reduce defensive responses so drastically (9). However, it is noteworthy that lesions in the PMD have a minimal effect on nonpredator threat stimuli, such as elevated plus-maze and postshock contextual cues (10), reinforcing the idea that different threats are integrated by distinct paths.

The predator-responsive medial hypothalamic circuit receives inputs from two amygdalar paths that integrate predator-related cues (Figure 1). The first is related to predator odor, processed by prey species in the accessory olfactory bulb, and transmitted to the medial amygdalar nucleus $(6,11)$. Rats exposed to cat odor show substantial activation of this nucleus, particularly in its posteroventral part (11). In line with this view, rats with cytotoxic lesions in the medial nucleus, but not in the central nucleus, exhibited a significant reduction in unconditioned fear responses to a live cat or its odor $(4,5)$. The second amygdalar path related to predator detection comprises the lateral and posterior basomedial amygdalar nuclei, known to receive inputs from the medial amygdala as well as from visual and auditory association areas, and is likely to integrate a wealth of predator-derived cues, from olfactory to non-olfactory ones (6). Cytotoxic lesions of these amygdalar sites have also been shown to reduce unconditioned defensive responses during exposure to a live predator (5). Both amygdalar paths target the predator-responsive medial hypothalamic circuit mostly by projecting to the dorsomedial part of the ventromedial nucleus.

Moreover, the predator-responsive medial hypothalamic circuit is also influenced by contextual cues from the environment, mostly via projections from a hippocampal-septal path to the anterior hypothalamic nucleus (Figure 1) (6). In fact, the degree of familiarity with a certain environment critically influences anti-predatory responses, and animals seem to be particularly responsive to predator threats in well-known environments (12).

The predator-responsive medial hypothalamic circuit seems to be preserved across species, and at least part of it is also present in humans and has been shown to organize fear responses. Functional magnetic resonance imaging (fMRI) studies in human participants have shown that video clips of threatening actions were able to induce a clear activation in the posterior half of the medial hypothalamus (13). This finding is particularly revealing, showing that, in humans, the hypothalamus is particularly responsive to psychological threats. Moreover, findings obtained from an awake patient undergoing bilateral implantation of deep brain stimulation electrodes into the hypothalamus have shown that the dorsomedial part of the ventromedial hypothalamic nucleus (VMHdm) presented the lowest threshold to induce panic attacks (14). Similar to what has been found for rodents during predatory exposure, in humans, the VMHdm may be thought to be part of a circuit that organizes complex active programs to support impending death situations, such as exposure to a war zone or gun threatening. Therefore, like predatory threats in rodents, psychological threats in humans seem to engage an analogous medial hypothalamic circuit, which is likely to have a large impact on fear responses, and perhaps on fear memory processing, as will be discussed for rodents.

Social threats, such as confrontations with a dominant male, represent a common fearful situation for the species, and have been shown to be integrated by elements of the social/reproductive medial hypothalamic circuit (including the medial preoptic area, the ventrolateral part of the ventromedial nucleus (VMHvm) and the ventral premamillary nucleus) and the dorsomedial part of the PMD nucleus 
(Figure 2) (7). Links between the social/reproductive medial hypothalamic circuit and the dorsomedial part of the PMD may be mediated by the subfornical region of the lateral hypothalamic area (15). Of particular interest in this regard is the fact that PMD-lesioned intruders lost passive defensive postures, like freezing and the stereotyped sustained on-the-back position, after resident departure and did not try to escape from the resident (7).

As shown in Figure 2, the social/reproductive medial hypothalamic circuit is dominated by pheromonal information largely relayed through specific parts of the medial amygdalar nucleus (i.e., its anterodorsal and posterodorsal parts) (8). In addition to social defense, this medial hypothalamic circuit also organizes other social behaviors (like social aggression) and reproductive behaviors (like sexual and maternal behaviors). In his analysis of the reproductive instinct, Tinbergen (16) placed fighting between conspecific opponents to establish a territory at the top of a hierarchy, followed by nest building, mating, and parental behavior. Therefore, a challenging question is to understand how these different responses can be prioritized and at the same time organized in the same circuit. Specific pheromonal cues are certainly critical to drive different social and reproductive behaviors. Interestingly, studies using immediate early gene analysis and single unit recordings from the ventrolateral $\mathrm{VMH}(\mathrm{VMHvl})$ during social interactions revealed overlapping but distinct neuronal subpopulations involved in fighting and mating, where neurons activated during attack were inhibited during mating, suggesting a potential neural substrate for competition between these

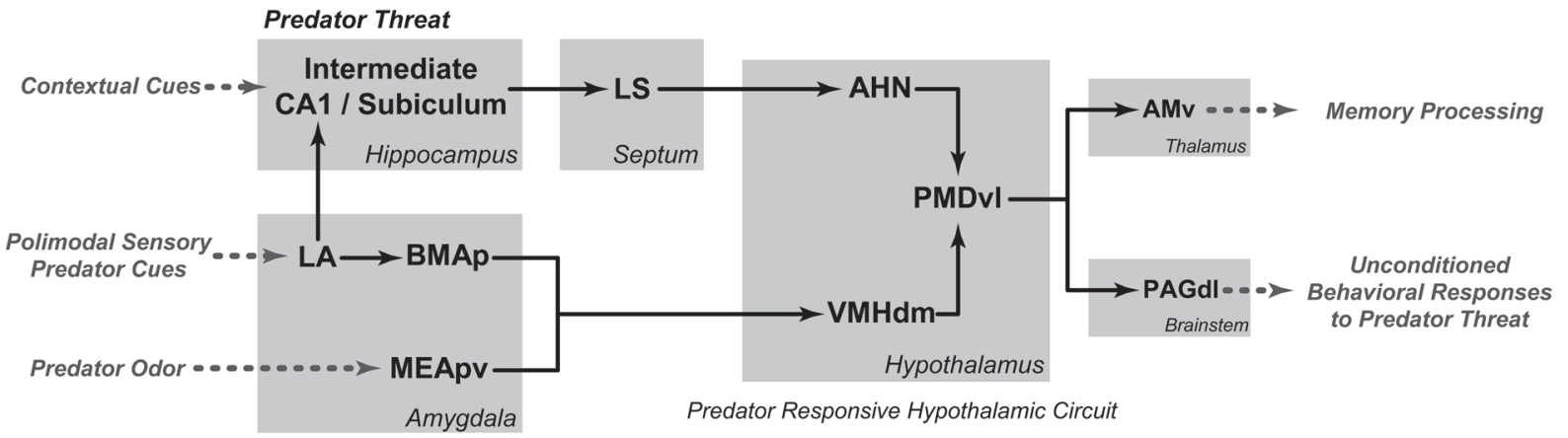

Figure 1. Schematic diagram showing the putative brain systems involved in processing predatory threats and organizing unconditioned defensive responses. AHN = anterior hypothalamic nucleus; $\mathrm{AMv}=$ anteromedial thalamic nucleus, ventral part; $\mathrm{BMAp}=$ basomedial amygdalar nucleus, posterior part; CA1 = field CA1, Ammon's horn; LA = lateral amygdalar nucleus; LS = lateral septal nucleus; MEApv = medial amygdalar nucleus, posteroventral part; PAGdl = periaqueductal gray, dorsolateral part; PMDvl = dorsal premamillary nucleus, ventrolateral part; VMHdm = ventromedial hypothalamic nucleus, dorsomedial part. This figure is a modified version of figure 8 of Ref. 9.

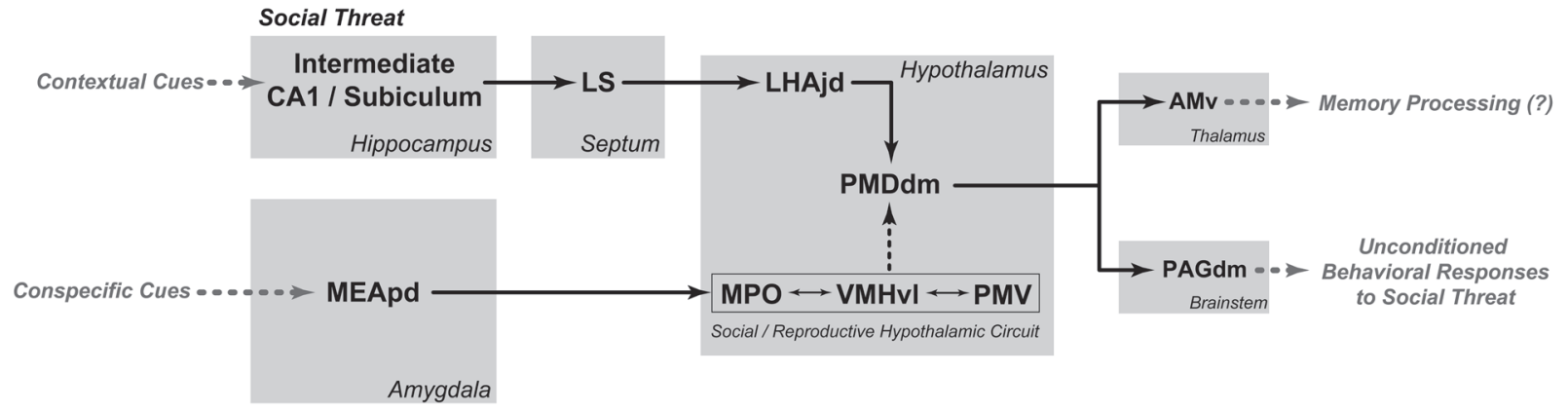

Figure 2. Schematic diagram showing the putative brain systems involved in processing social threats and organizing unconditioned defensive responses. AMv = anteromedial thalamic nucleus, ventral part; CA1 = field CA1, Ammon's horn; LHAjd = lateral hypothalamic area, juxtadorsomedial region; LS = lateral septal nucleus; MEApd = medial amygdalar nucleus, posterodorsal part; MPO = medial preoptic area; PAGdm = periaqueductal gray, dorsomedial part; PMDdm = dorsal premamillary nucleus, dorsomedial part; PMV = ventral premamillary nucleus; $\mathrm{VMHvI}=$ ventromedial hypothalamic nucleus, ventrolateral part. 
opposite social behaviors (17). Moreover, elements of the social/reproductive medial hypothalamic circuit are highly responsive to sexual steroids, which are known to influence a number of social and reproductive behaviors, as in the case of testosterone that can facilitate social aggression.

Possible genes controlling the wiring of these parallel amygdalar-hypothalamic paths to process predatory and social threats have been reported. It has been shown that different LIM homeodomain transcription factors mark neurons in different parts of the medial amygdala; and particularly striking was the finding that Lhx6, expressed in both the posterodorsal part of the medial amygdalar nucleus and in the principal nucleus of the bed nucleus of the stria terminalis (BST), delineates the projections to the elements of the social/reproductive medial hypothalamic circuit (18).

\section{The PAG and its role in the expression of fear responses}

The parallel paths processing different classes of threats (i.e., conditioning to painful stimuli, predators and aggressive dominant conspecifics) have a common target - the PAG critical for the organization of all kinds of fear responses.

Freezing is the typical behavioral response seen in fear conditioning to painful stimuli, a response that depends on the integrity of the central amygdalar nucleus (CEA) $(1,19)$. In the CEA, the lateral part (CEAI) has an inhibitory influence on the medial part (CEAm), and aversive CS suppresses this inhibition on the CEAm, which, via projections to the ventrolateral part of the PAG (PAGvl), drives the conditioned freezing (3). The PAGvl is critical for the expression of conditioned fear responses, including freezing, vocalization and conditioned analgesia. Lesions of the PAGvl reduced freezing responses to neutral stimuli associated with footshock (20). This system has been shown to be preserved across species, and human neuroimaging studies using fear conditioning to painful stimuli have reported increased blood oxygen level-dependent signals in the PAGvl during fear expression, which is maximal at high levels of threat imminence (21).

As shown in Figure 1, the predator-responsive medial hypothalamic circuit preferentially targets the dorsolateral part of the PAG (PAGdl). Stimulation of the PAGdl elicits species-specific autonomic and behavioral defensive responses in cats and rats, as well as feelings of fear, impending death and apprehensive avoidance in humans (22-24). The kind of fear responses to a predator-related cue depends on its degree of ambiguity for signaling the predator presence. Thus, fear responses to the actual predator include mostly freezing and, depending on the proximity of the predator, also flight responses when the predator is nearby (12). On the other hand, fear responses to a more ambiguous threat, like predator odor, are characterized by risk assessment responses, including a careful scanning of the environment in the crouch position (crouch sniffing) and attempts to approach the threatening stimulus by stretching the body (stretch postures) (25). The PAGdl presents a characteristic Fos up-regulation in response to predatorrelated cues, which also varies depending on the ambiguity of the stimulus. Thus, depending on the degree of PAGdl activation, the behavioral outcome may be flight/freezing responses to stronger PAGdl activation, or risk assessment in situations of higher ambiguity and weaker PAGdl activation (9). In line with this view, NMDA cytotoxic lesions in the dorsal PAG have been shown to block flight/freezing and risk assessment responses to predator cues (26).

In the context of social threats by dominant conspecifics, the dorsomedial part of the dorsal premamillary nucleus (PMDdm) serves as an important interface between the social/reproductive medial hypothalamic circuit and the PAG (Figure 2). The PAG Fos activation pattern in response to a dominant conspecific matches the axonal projection pattern from the PMDdm, and was found in the dorsomedial and lateral parts of the PAG (7). Preliminary findings from our laboratory suggest that the dorsomedial, but not the lateral, PAG seems to be critical for the expression of both passive (i.e., freezing and the typical on-the-back position maintained after the resident leaves them alone) and active (i.e., upright position with sparse boxing and dashing away from the resident) forms of social defensive responses.

\section{Cortical-hippocampal-amygdalar paths involved in fear memory}

Fear memory related to different kinds of threats also appears to share common cortical-hippocampal-amygdalar paths. Fear learning has been mostly investigated with classical Pavlovian fear conditioning paradigms, using the association of a US (usually a painful stimulus) and a neutral CS. In this paradigm, the amygdala was found to be central for the CS to acquire aversiveness, while the hippocampus primarily processes information associated with the fear context $(1,19)$. As pointed out above, the lateral nucleus of the amygdala is thought of as the primary site where associations between the conditioned stimulus CS and the US are formed and stored, and known as a critical site for fear memory, and the hippocampus has been involved in Pavlovian fear conditioning to contextual cues. Studies using classical fear conditioning have also shown that a number of cortical fields influence amygdalar- and hippocampal-dependent learning, and particular emphasis has been given to the anterior cingulate (ACA), retrosplenial (RSP) and postrhinal (POR) areas. Chemical or electrical stimulation of the ACA has been used as a useful US sufficient to support fear learning, whereas pharmacological inhibition or antagonism of glutamate receptors in the ACA can retard or prevent such learning for both contextual and auditory fear memory $(27,28)$. This effect is likely to be mediated by either direct or indirect projections to the 
lateral amygdalar nucleus and hippocampus. Indirect paths appear to involve the RSP, where electrolytic or chemical damage either before or immediately after training impaired the expression of contextual fear but not of tone-specific fear $(29,30)$. The RSP is thought to influence the contextual fear processing through its projections to the POR, another region shown to be involved in contextual fear conditioning, seemingly mediated by the POR projections to the hippocampal formation and lateral amygdala (31). Therefore, a pathway involving the ACA, RSP and POR forms part of a cortical circuit influencing fear memory in the amygdala and hippocampus.

Animals rapidly acquire contextual conditioned defensive responses to an environment where they had previously encountered a predator, or the odor of a predator (32). Thus, long-term risk assessment, as well as freezing and avoidance, are seen in the area where a live predator or its skin/fur odor had been encountered. Similar to what was discussed for Pavlovian fear conditioning to painful stimuli, the hippocampus and the lateral amygdalar nucleus play a key role in fear memory related to predator threats. In line with this view, findings from Blanchard's (32) laboratory indicate that ventral hippocampal lesions (including intermediate and ventral regions of field CA1 and subiculum) significantly reduced conditioned defensive behaviors during re-exposure to the context associated with either direct exposure to the cat or exposure to its odor (33). Moreover, it has been shown that the basolateral amygdalar complex is involved in contextual fear memory consolidation in response to a predator-related environment. Lesions of the posterior basomedial and lateral amygdalar nuclei yielded an important reduction in the contextual fear responses to a live cat (5); and studies using lesion or pharmacological inhibition of the basolateral amygdalar complex immediately after exposure to cat odor have shown a significant reduction in the contextual defensive responses in a conditioned fear test (34).

As shown in Figure 3, in contrast to Pavlovian fear conditioning to painful stimuli, predator threats are processed by the predator-responsive medial hypothalamic circuit, where the PMDvl is the most responsive site to predatory threats. It has been shown that pharmacological blockade of the PMD targeting either the NMDA-receptors or the beta-adrenoceptors, markedly influenced associative mechanisms linking predatory threats to the related context $(25,35)$. The PMD provides a robust branched projection to the PAG and thalamus (6). In the thalamus, the PMD provides a dense projection to the ventral part of the anteromedial thalamic nucleus (AMv). Bilateral AMv lesions did not alter the unconditioned responses to a live cat, but completely blocked the contextual conditioned defensive responses (36). The AMv role in contextual fear should involve its cortical projections to the anterior cingulate and retrosplenial areas (37), which, as discussed above, form part of a cortical circuit influencing fear memory in the amygdala and hippocampus (Figure 3). Therefore, fear conditioning to both painful stimuli and predator threats may share common cortico-hippocampal-amygdalar paths. At this point, it would be important to determine whether conditioning to painful stimuli and predator threats shares

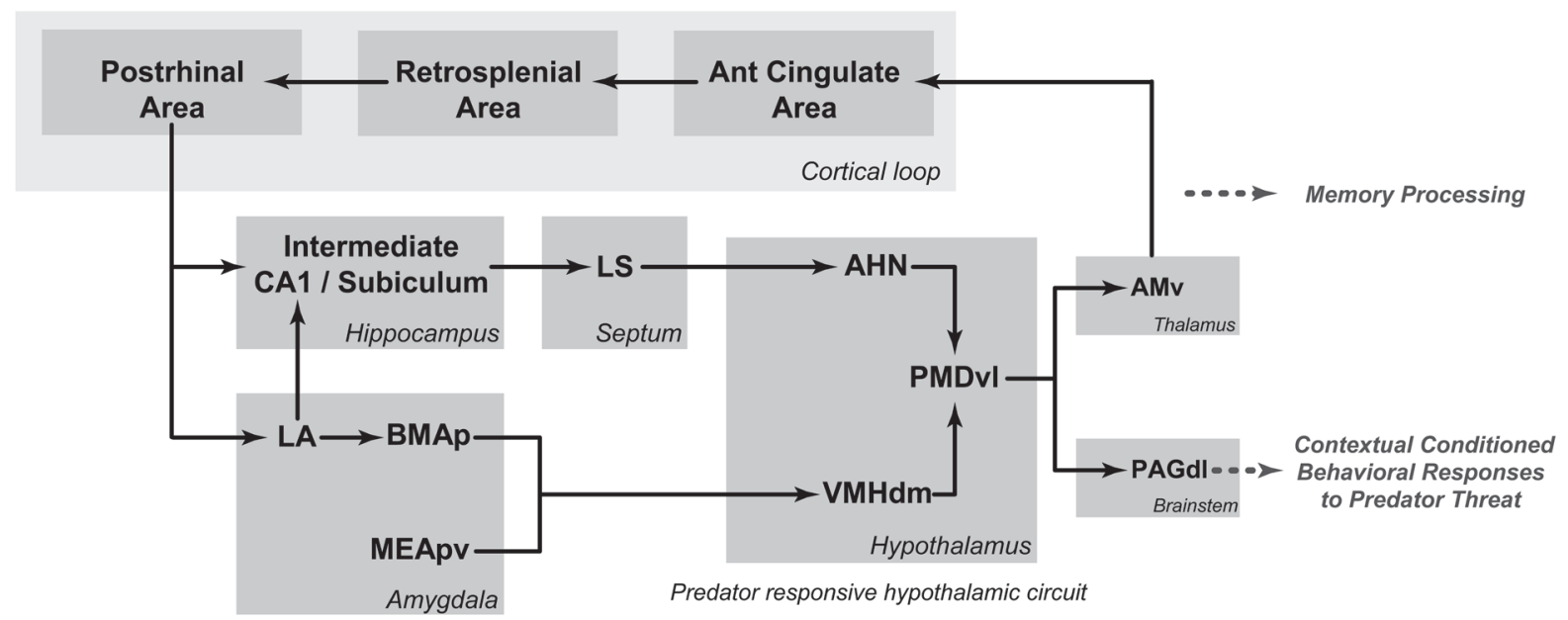

Figure 3. Schematic diagram showing the putative circuits involved in processing fear memory to predatory threats and organizing contextual conditioned defensive responses. $\mathrm{AHN}=$ anterior hypothalamic nucleus; $\mathrm{AMv}=$ anteromedial thalamic nucleus, ventral part; BMAp = basomedial amygdalar nucleus, posterior part; CA1 = field CA1, Ammon's horn; LA = lateral amygdalar nucleus; LS = lateral septal nucleus; MEApv = medial amygdalar nucleus, posteroventral part; PAGdl = periaqueductal gray, dorsolateral part; PMDvl = dorsal premamillary nucleus, ventrolateral part; $\mathrm{VMHdm}=$ ventromedial hypothalamic nucleus, dorsomedial part. 
the same properties in terms of extinction, reconsolidation and molecular basis of the fear memory.

As previously noted, lesions of the CEA do not affect the contextual fear response to a predator threat, which relies on the predator-responsive medial hypothalamic circuit and the PAGdl. As shown in Figure 3, the ventral hippocampus and lateral amygdalar nucleus provide projection paths to the anterior hypothalamic nucleus and the dorsomedial part of the ventromedial nucleus, respectively, which are likely to integrate contextual fear memory information. Pharmacological inactivation of the PMD has been shown to block contextual fear responses to predator threats (9).

\section{Concluding remarks}

Ethologically based models have taught us a great deal about the neural systems underlying fear and anxiety. First, they have shown that different threats mobilize distinct neural systems, challenging the current view of a unitary core circuit for fear processing centered on the central amygdala. Of particular interest, ethologically based models have shown that the medial hypothalamus is a critical spot

\section{References}

1. LeDoux JE. Emotion circuits in the brain. Annu Rev Neurosci 2000; 23: 155-184.

2. Blair HT, Schafe GE, Bauer EP, Rodrigues SM, LeDoux JE. Synaptic plasticity in the lateral amygdala: a cellular hypothesis of fear conditioning. Learn Mem 2001; 8: 229-242.

3. Ciocchi S, Herry C, Grenier F, Wolff SB, Letzkus JJ, Vlachos $\mathrm{I}$, et al. Encoding of conditioned fear in central amygdala inhibitory circuits. Nature 2010; 468: 277-282.

4. Li Cl, Maglinao TL, Takahashi LK. Medial amygdala modulation of predator odor-induced unconditioned fear in the rat. Behav Neurosci 2004; 118: 324-332.

5. Martinez RC, Carvalho-Netto EF, Ribeiro-Barbosa ER, Baldo MV, Canteras NS. Amygdalar roles during exposure to a live predator and to a predator-associated context. Neuroscience 2011; 172: 314-328.

6. Canteras NS. The medial hypothalamic defensive system: hodological organization and functional implications. Pharmacol Biochem Behav 2002; 71: 481-491.

7. Motta SC, Goto M, Gouveia FV, Baldo MV, Canteras NS, Swanson LW. Dissecting the brain's fear system reveals the hypothalamus is critical for responding in subordinate conspecific intruders. Proc Natl Acad Sci U S A 2009; 106: 4870-4875.

8. Swanson LW. Cerebral hemisphere regulation of motivated behavior. Brain Res 2000; 886: 113-164.

9. Cezario AF, Ribeiro-Barbosa ER, Baldo MV, Canteras NS. Hypothalamic sites responding to predator threats - the role of the dorsal premammillary nucleus in unconditioned and conditioned antipredatory defensive behavior. Eur J Neurosci 2008; 28: 1003-1015.

10. Blanchard DC, Li Cl, Hubbard D, Markham CM, Yang M, Takahashi LK, et al. Dorsal premammillary nucleus differ- for the integration of predator cues and social threats, the kind of roles that fit well with the general hypothalamic tasks in ensuring survival and reproductive fitness.

Another important view that has emerged from the present analysis is that the parallel paths processing different classes of threats (i.e., painful stimuli, predators and aggressive dominant conspecifics) have a common target - the PAG, which is critical for the expression of fear responses to all kinds of fear.

Finally, despite the parallel processing of the different classes of threats, common cortical-hippocampal-amygdalar paths seem to be engaged in the fear conditioning to painful stimuli, to predators, and perhaps to aggressive dominant conspecifics as well.

\section{Acknowledgments}

Research supported by FAPESP (grants \#05/59286-4 and \#09/53390-5 to N.S. Canteras and S.R. Mota-Ortiz) and CNPq (N.S. Canteras). S.C. Motta was supported by a FAPESP fellowship (\#10/05905-3). entially modulates defensive behaviors induced by different threat stimuli in rats. Neurosci Lett 2003; 345: 145-148.

11. Dielenberg RA, Hunt GE, McGregor IS. "When a rat smells a cat": the distribution of Fos immunoreactivity in rat brain following exposure to a predatory odor. Neuroscience 2001; 104: 1085-1097.

12. Ribeiro-Barbosa ER, Canteras NS, Cezario AF, Blanchard RJ, Blanchard DC. An alternative experimental procedure for studying predator-related defensive responses. Neurosci Biobehav Rev 2005; 29: 1255-1263.

13. Pichon S, de Gelder B, Grezes J. Threat prompts defensive brain responses independently of attentional control. Cereb Cortex 2012; 22: 274-285.

14. Wilent WB, Oh MY, Buetefisch CM, Bailes JE, Cantella D, Angle $C$, et al. Induction of panic attack by stimulation of the ventromedial hypothalamus. J Neurosurg 2010; 112: 12951298.

15. Goto M, Canteras NS, Burns G, Swanson LW. Projections from the subfornical region of the lateral hypothalamic area. J Comp Neurol 2005; 493: 412-438.

16. Tinbergen N. The study of instinct. London, Oxford University Press: 1951.

17. Lin D, Boyle MP, Dollar P, Lee H, Lein ES, Perona P, et al. Functional identification of an aggression locus in the mouse hypothalamus. Nature 2011; 470: 221-226.

18. Choi GB, Dong HW, Murphy AJ, Valenzuela DM, Yancopoulos GD, Swanson LW, et al. Lhx6 delineates a pathway mediating innate reproductive behaviors from the amygdala to the hypothalamus. Neuron 2005; 46: 647-660.

19. Maren S. Neurobiology of Pavlovian fear conditioning. Annu Rev Neurosci 2001; 24: 897-931.

20. Vianna DM, Landeira-Fernandez J, Brandão ML. Dorsolat- 
eral and ventral regions of the periaqueductal gray matter are involved in distinct types of fear. Neurosci Biobehav Rev 2001; 25: 711-719.

21. Mobbs D, Petrovic P, Marchant JL, Hassabis D, Weiskopf N, Seymour B, et al. When fear is near: threat imminence elicits prefrontal-periaqueductal gray shifts in humans. Science 2007; 317: 1079-1083.

22. Hunsperger RW. [Affective reaction from electric stimulation of brain stem in cats]. Helv Physiol Pharmacol Acta 1956; 14: 70-92.

23. Nashold BS Jr, Wilson WP, Slaughter DG. Sensations evoked by stimulation in the midbrain of man. J Neurosurg 1969; 30: 14-24.

24. Bittencourt AS, Carobrez AP, Zamprogno LP, Tufik S, Schenberg LC. Organization of single components of defensive behaviors within distinct columns of periaqueductal gray matter of the rat: role of $\mathrm{N}$-methyl-D-aspartic acid glutamate receptors. Neuroscience 2004; 125: 71-89.

25. Canteras NS, Kroon JA, Do-Monte FH, Pavesi E, Carobrez AP. Sensing danger through the olfactory system: the role of the hypothalamic dorsal premammillary nucleus. Neurosci Biobehav Rev 2008; 32: 1228-1235.

26. Sukikara MH, Mota-Ortiz SR, Baldo MV, Felicio LF, Canteras NS. The periaqueductal gray and its potential role in maternal behavior inhibition in response to predatory threats. Behav Brain Res 2010; 209: 226-233.

27. Tang J, Ko S, Ding HK, Qiu CS, Calejesan AA, Zhuo M. Pavlovian fear memory induced by activation in the anterior cingulate cortex. Mol Pain 2005; $1: 6$.

28. Bissiere S, Plachta N, Hoyer D, McAllister KH, Olpe HR, Grace AA, et al. The rostral anterior cingulate cortex modulates the efficiency of amygdala-dependent fear learning.
Biol Psychiatry 2008; 63: 821-831.

29. Keene CS, Bucci DJ. Contributions of the retrosplenial and posterior parietal cortices to cue-specific and contextual fear conditioning. Behav Neurosci 2008; 122: 89-97.

30. Keene CS, Bucci DJ. Neurotoxic lesions of retrosplenial cortex disrupt signaled and unsignaled contextual fear conditioning. Behav Neurosci 2008; 122: 1070-1077.

31. Burwell RD, Saddoris MP, Bucci DJ, Wiig KA. Corticohippocampal contributions to spatial and contextual learning. $J$ Neurosci 2004; 24: 3826-3836.

32. Blanchard RJ, Yang M, Li Cl, Gervacio A, Blanchard DC. Cue and context conditioning of defensive behaviors to cat odor stimuli. Neurosci Biobehav Rev 2001; 25: 587-595.

33. Pentkowski NS, Blanchard DC, Lever C, Litvin Y, Blanchard RJ. Effects of lesions to the dorsal and ventral hippocampus on defensive behaviors in rats. Eur J Neurosci 2006; 23: 2185-2196.

34. Takahashi LK, Hubbard DT, Lee I, Dar Y, Sipes SM. Predator odor-induced conditioned fear involves the basolateral and medial amygdala. Behav Neurosci 2007; 121: 100-110.

35. Do Monte FH, Canteras NS, Fernandes D, Assreuy J, Carobrez AP. New perspectives on beta-adrenergic mediation of innate and learned fear responses to predator odor. $J$ Neurosci 2008; 28: 13296-13302.

36. Carvalho-Netto EF, Martinez RC, Baldo MV, Canteras NS. Evidence for the thalamic targets of the medial hypothalamic defensive system mediating emotional memory to predatory threats. Neurobiol Learn Mem 2010; 93: 479-486.

37. van Groen T, Kadish I, Wyss JM. Efferent connections of the anteromedial nucleus of the thalamus of the rat. Brain Res Brain Res Rev 1999; 30: 1-26. 